Tiankun Zhao*, Peng Wang, Mingyu Ji, Shanjia Li, Mingjun Yang and Xiuying Pu

\title{
The crystal structure of $1,1^{\prime}-(((2$ (dimethylamino) ethyl)azanediyl)bis(methylene)) bis(naphthalen- 2-olato- $\left.\mathrm{K}^{4} N, N^{\prime}, O, O^{\prime}\right)$-(pyridine-2,6-dicarboxylato- $\left.N, O, O^{\prime}\right)$ - titanium(IV) - dichloromethane (2/1), $\mathrm{C}_{33} \mathrm{H}_{29} \mathrm{~N}_{3} \mathrm{O}_{6} \mathrm{Ti}$
}
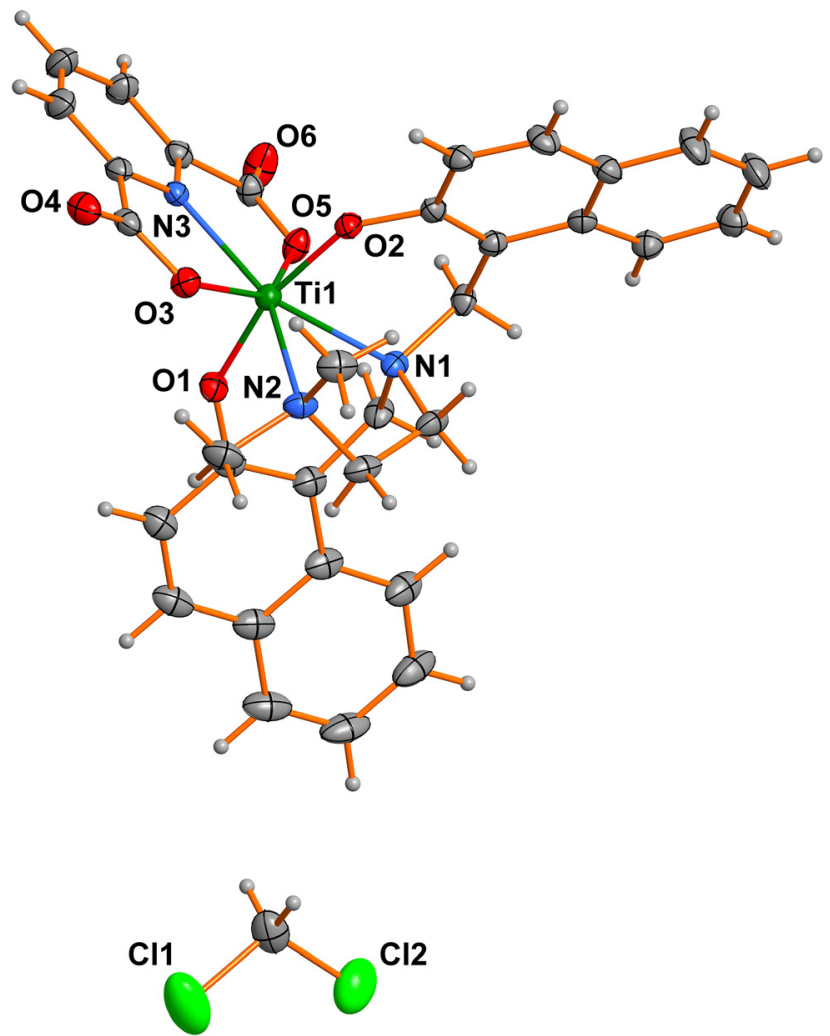

https://doi.org/10.1515/ncrs-2021-0182

Received May 12, 2021; accepted June 8, 2001; published online July 6, 2021

\begin{abstract} $\mathrm{C}_{33} \mathrm{H}_{29} \mathrm{~N}_{3} \mathrm{O}_{6} \mathrm{Ti}$, monoclinic, $C 2 / c$ (no. 15), $a=27.4022(15) \AA$, $b=14.0345(7) \AA, c=17.0047(10) \AA, \beta=116.009(3)^{\circ}$, $V=5877.3(6) \AA^{3}, Z=8, R_{g t}(F)=0.0369, w R_{r e f}\left(F^{2}\right)=0.1125$, $T=100(2) \mathrm{K}$.
\end{abstract}

*Corresponding author: Tiankun Zhao, College of Life Science and Engineering, Lanzhou University of Technology, Lanzhou, 730050, Gansu, P. R. China, E-mail: zhaotiankun2006@163.com. https:// orcid.org/0000-0001-6675-5586

Peng Wang, Mingyu Ji, Shanjia Li, Mingjun Yang and Xiuying Pu, College of Life Science and Engineering, Lanzhou University of Technology, Lanzhou, 730050, Gansu, P. R. China
Table 1: Data collection and handling.

\begin{tabular}{|c|c|}
\hline Crystal: & Orange block \\
\hline Size: & $0.12 \times 0.10 \times 0.10 \mathrm{~mm}$ \\
\hline Wavelength: & Ga $K \alpha$ radiation $(1.34138 \AA$ ) \\
\hline$\mu:$ & $2.51 \mathrm{~mm}^{-1}$ \\
\hline Diffractometer, scan mode: & Bruker APEX-II, $\varphi$ and $\omega$ \\
\hline$\theta_{\max }$, completeness: & $57.1^{\circ},>99 \%$ \\
\hline$N(h k l)_{\text {measured }}, N(h k l)_{\text {unique }}, R_{\text {int }}:$ & $38312,6027,0.052$ \\
\hline Criterion for $I_{\mathrm{obs}}, N\left(h k l_{\mathrm{gt}}\right.$ : & $I_{\text {obs }}>2 \sigma\left(I_{\text {obs }}\right), 5177$ \\
\hline$N(\text { param })_{\text {refined: }}$ & 418 \\
\hline Programs: & Bruker [1], SHELX [2] \\
\hline
\end{tabular}

CCDC no.: 2082174

The molecular structure is shown in the figure. Table 1 contains crystallographic data and Table 2 contains the list of the atoms including atomic coordinates and displacement parameters.

\section{Source of material}

The ONON ligand $\mathrm{L}_{1}$ : (1,1'-((2-(dimethyl-amino)ethyl) azanediyl)bis (methylene))bis(naphthalen-2-ol)) was prepared following the literature reported method by prolonging the reaction time from 2 to $6 \mathrm{~h}$ [3]. The corresponding Ti(IV) complex was synthesized according to lit. Under a nitrogen atmosphere, $\mathrm{L}_{1}(1 \mathrm{mmol}, 400 \mathrm{mg})$ was dissolved in anhydrous THF $(15 \mathrm{~mL}), \mathrm{Ti}\left(\mathrm{O}^{\mathrm{i}} \mathrm{Pr}\right)_{4}$ $(0.95 \mathrm{mmol}, 0.28 \mathrm{~mL})$ was then added to the solution. The reaction was heated to $50^{\circ} \mathrm{C}$ and kept stirring for $2 \mathrm{~h}$, Dipic (pyridine-2,6-dicarboxylic acid) $(0.95 \mathrm{mmol}$, $158.8 \mathrm{mg}$ ) was added and the reaction was left stirring for another $10 \mathrm{~h}$ and monitored by TLC. When the reaction was complete, $\left[\mathrm{L}_{1} \mathrm{Ti}^{(\mathrm{IV})}\right.$ (Dipic)] was separated by flash column chromatography $\left(\mathrm{CH}_{2} \mathrm{Cl}_{2} / \mathrm{CH}_{3} \mathrm{OH}=30 / 1\right)$ to obtain a red solid $(0.80 \mathrm{mmol}, 492.1 \mathrm{mg}, 85 \%)$. Suitable crystals for X-ray diffraction measurements were obtained by slow diffusion of $n$-hexane to a dichloromethane solution of $\left[\mathrm{L}_{1} \mathrm{Ti}^{(\mathrm{IV})}\right.$ (Dipic)] at room temperature. 
Table 2: Fractional atomic coordinates and isotropic or equivalent isotropic displacement parameters $\left(\AA^{2}\right)$.

\begin{tabular}{|c|c|c|c|c|}
\hline Atom & $x$ & $y$ & $z$ & $U_{\text {iso }} * / U_{\text {eq }}$ \\
\hline $\mathrm{C} 1$ & $0.60189(7)$ & $0.50113(13)$ & $0.58013(12)$ & $0.0233(4)$ \\
\hline $\mathrm{C} 2$ & $0.58945(7)$ & 0.51514 (13) & $0.64972(12)$ & $0.0216(4)$ \\
\hline C3 & $0.54729(7)$ & $0.46373(14)$ & 0.65755 (13) & $0.0257(4)$ \\
\hline H3 & 0.539570 & 0.474178 & 0.706156 & $0.031^{*}$ \\
\hline $\mathrm{C} 4$ & $0.51786(7)$ & 0.39903 (14) & 0.59457 (13) & 0.0285 (4) \\
\hline $\mathrm{H} 4$ & 0.489233 & 0.365464 & 0.599549 & $0.034^{*}$ \\
\hline C5 & $0.52879(7)$ & $0.38076(14)$ & $0.52254(13)$ & $0.0274(4)$ \\
\hline $\mathrm{C} 6$ & $0.57149(7)$ & $0.43256(14)$ & $0.51411(13)$ & 0.0255 (4) \\
\hline $\mathrm{C} 7$ & $0.58010(7)$ & $0.41355(15)$ & $0.43924(14)$ & $0.0315(5)$ \\
\hline $\mathrm{H} 7$ & 0.607797 & 0.447275 & 0.431655 & $0.038^{\star}$ \\
\hline C8 & 0.54954 (8) & $0.34773(16)$ & $0.37731(15)$ & $0.0379(5)$ \\
\hline $\mathrm{H} 8$ & 0.555907 & 0.337312 & 0.327385 & $0.046^{*}$ \\
\hline C9 & $0.50878(8)$ & $0.29568(16)$ & 0.38777 (16) & $0.0397(6)$ \\
\hline H9 & 0.488380 & 0.248968 & 0.345741 & $0.048^{\star}$ \\
\hline C10 & $0.49865(8)$ & 0.31234 (14) & 0.45804 (15) & $0.0352(5)$ \\
\hline $\mathrm{H} 10$ & 0.470790 & 0.277395 & 0.464063 & $0.042^{\star}$ \\
\hline C11 & $0.64373(7)$ & $0.56345(14)$ & $0.57011(12)$ & $0.0231(4)$ \\
\hline $\mathrm{H} 11 \mathrm{~A}$ & 0.651376 & 0.536388 & 0.522901 & $0.028^{\star}$ \\
\hline $\mathrm{H} 11 \mathrm{~B}$ & 0.627497 & 0.627221 & 0.550497 & $0.028^{\star}$ \\
\hline C12 & $0.72841(7)$ & $0.64323(13)$ & $0.62014(11)$ & $0.0203(4)$ \\
\hline $\mathrm{H} 12 \mathrm{~A}$ & 0.707714 & 0.703407 & 0.600574 & $0.024^{*}$ \\
\hline $\mathrm{H} 12 \mathrm{~B}$ & 0.731116 & 0.614728 & 0.568945 & $0.024^{*}$ \\
\hline C13 & $0.78488(7)$ & $0.66628(12)$ & $0.68869(12)$ & 0.0185 (4) \\
\hline C14 & $0.79433(7)$ & $0.68331(12)$ & $0.77415(12)$ & 0.0185 (4) \\
\hline C15 & $0.84694(7)$ & 0.70365 (13) & $0.84046(12)$ & $0.0215(4)$ \\
\hline $\mathrm{H} 15$ & 0.852252 & 0.714900 & 0.898766 & $0.026^{*}$ \\
\hline C16 & $0.88981(7)$ & 0.70705 (14) & 0.82051 (13) & $0.0254(4)$ \\
\hline $\mathrm{H} 16$ & 0.925271 & 0.718108 & 0.865577 & $0.030 *$ \\
\hline C17 & $0.88219(7)$ & $0.69428(13)$ & $0.73334(12)$ & $0.0233(4)$ \\
\hline C18 & $0.82922(7)$ & $0.67450(12)$ & $0.66575(12)$ & $0.0186(4)$ \\
\hline C19 & $0.82341(7)$ & $0.66746(13)$ & $0.57918(12)$ & $0.0225(4)$ \\
\hline H19 & 0.788488 & 0.655293 & 0.533004 & $0.027^{\star}$ \\
\hline $\mathrm{C} 20$ & $0.86674(8)$ & $0.67768(14)$ & 0.55999 (13) & $0.0266(4)$ \\
\hline $\mathrm{H} 20$ & 0.861538 & 0.672721 & 0.501104 & $0.032^{\star}$ \\
\hline $\mathrm{C} 21$ & $0.91865(8)$ & $0.69541(16)$ & 0.62681 (14) & $0.0347(5)$ \\
\hline $\mathrm{H} 21$ & 0.948694 & 0.701718 & 0.613397 & $0.042^{\star}$ \\
\hline $\mathrm{C} 22$ & $0.92602(8)$ & $0.70360(16)$ & 0.71120 (14) & $0.0337(5)$ \\
\hline $\mathrm{H} 22$ & 0.961378 & 0.715836 & 0.756097 & $0.040^{*}$ \\
\hline $\mathrm{C} 23$ & $0.72777(7)$ & $0.48444(13)$ & $0.67352(13)$ & $0.0241(4)$ \\
\hline $\mathrm{H} 23 \mathrm{~A}$ & 0.767214 & 0.497935 & 0.702948 & $0.029^{\star}$ \\
\hline $\mathrm{H} 23 \mathrm{~B}$ & 0.720080 & 0.448072 & 0.619458 & 0.029 * \\
\hline C24 & $0.71249(7)$ & $0.42561(13)$ & 0.73265 (13) & $0.0262(4)$ \\
\hline $\mathrm{H} 24 \mathrm{~A}$ & 0.674471 & 0.403672 & 0.700276 & 0.031 * \\
\hline $\mathrm{H} 24 \mathrm{~B}$ & 0.736164 & 0.368694 & 0.752106 & $0.031^{*}$ \\
\hline $\mathrm{C} 25$ & $0.77619(7)$ & $0.48459(14)$ & 0.87507 (13) & $0.0281(4)$ \\
\hline $\mathrm{H} 25 \mathrm{~A}$ & 0.789033 & 0.419579 & 0.893957 & $0.042^{*}$ \\
\hline $\mathrm{H} 25 \mathrm{~B}$ & 0.779958 & 0.522532 & 0.925811 & $0.042^{\star}$ \\
\hline $\mathrm{H} 25 \mathrm{C}$ & 0.797847 & 0.513437 & 0.848288 & $0.042^{*}$ \\
\hline C26 & $0.68845(8)$ & $0.42931(14)$ & 0.85141 (14) & $0.0306(4)$ \\
\hline $\mathrm{H} 26 \mathrm{~A}$ & 0.649620 & 0.428229 & 0.811402 & $0.046^{*}$ \\
\hline $\mathrm{H} 26 \mathrm{~B}$ & 0.693933 & 0.461093 & 0.905982 & $0.046^{*}$ \\
\hline $\mathrm{H} 26 \mathrm{C}$ & 0.702170 & 0.363861 & 0.864108 & $0.046^{*}$ \\
\hline $\mathrm{C} 27$ & $0.66641(7)$ & $0.77403(13)$ & $0.89390(11)$ & $0.0197(4)$ \\
\hline C28 & $0.65133(8)$ & $0.85380(14)$ & 0.92549 (13) & $0.0274(4)$ \\
\hline H28 & 0.658098 & 0.857718 & 0.985179 & $0.033^{*}$ \\
\hline
\end{tabular}

Table 2: (continued)

\begin{tabular}{|c|c|c|c|c|}
\hline Atom & $x$ & $y$ & $z$ & $U_{\text {iso }} * / U_{\text {eq }}$ \\
\hline C29 & $0.62611(9)$ & $0.92803(14)$ & $0.86822(14)$ & $0.0316(5)$ \\
\hline $\mathrm{H} 29$ & 0.615201 & 0.983574 & 0.888247 & $0.038^{*}$ \\
\hline $\mathrm{C} 30$ & $0.61690(8)$ & 0.92068 (14) & $0.78133(13)$ & $0.0266(4)$ \\
\hline $\mathrm{H} 30$ & 0.599543 & 0.970676 & 0.741017 & $0.032^{*}$ \\
\hline C31 & $0.63362(7)$ & 0.83877 (13) & $0.75503(12)$ & $0.0195(4)$ \\
\hline C32 & $0.69347(7)$ & $0.68601(13)$ & $0.94366(12)$ & 0.0205 (4) \\
\hline C33 & $0.62923(7)$ & 0.81744 (13) & $0.66596(12)$ & $0.0216(4)$ \\
\hline $\mathrm{N} 1$ & $0.69711(5)$ & $0.57630(10)$ & $0.65008(10)$ & $0.0192(3)$ \\
\hline N2 & $0.71818(6)$ & $0.48192(11)$ & $0.81012(10)$ & 0.0225 (3) \\
\hline N3 & $0.65750(5)$ & $0.76734(10)$ & $0.81067(9)$ & $0.0169(3)$ \\
\hline 01 & $0.61798(5)$ & $0.57717(9)$ & $0.71411(8)$ & 0.0217 (3) \\
\hline 02 & $0.75358(5)$ & $0.68096(9)$ & $0.79766(8)$ & 0.0197 (3) \\
\hline 03 & $0.69884(5)$ & $0.62172(9)$ & $0.89296(8)$ & $0.0218(3)$ \\
\hline 04 & $0.70781(5)$ & $0.67830(10)$ & $1.02224(8)$ & $0.0276(3)$ \\
\hline 05 & $0.65263(5)$ & $0.73842(9)$ & $0.66427(8)$ & $0.0236(3)$ \\
\hline 06 & $0.60584(6)$ & $0.87121(10)$ & $0.60429(9)$ & 0.0353 (4) \\
\hline Ti1 & $0.68385(2)$ & $0.63935(2)$ & $0.76620(2)$ & $0.01642(11)$ \\
\hline $\mathrm{C} 1 \mathrm{~S}^{\mathrm{a}}$ & 0.51005 (19) & $0.0260(3)$ & $0.5073(4)$ & $0.0388(11)$ \\
\hline $\mathrm{H} 1 \mathrm{SA}^{\mathrm{a}}$ & 0.494042 & 0.090634 & 0.497869 & $0.047^{*}$ \\
\hline $\mathrm{H} 1 \mathrm{SB}^{\mathrm{a}}$ & 0.548342 & 0.030660 & 0.551686 & $0.047^{*}$ \\
\hline $\mathrm{Cl}^{\mathrm{a}}$ & $0.47435(7)$ & $-0.04917(14)$ & $0.54657(14)$ & $0.0779(5)$ \\
\hline $\mathrm{Cl}^{\mathrm{a}}$ & $0.50780(5)$ & $-0.01697(13)$ & $0.40881(9)$ & $0.0598(4)$ \\
\hline
\end{tabular}

\section{Experimental details}

$\mathrm{H}$ atoms bonded to $\mathrm{C}$ atoms were positioned geometrically with $\mathrm{C}-\mathrm{H}=0.95 \AA$ (aromatic), $0.99 \AA$ (methylene) and $0.98 \AA$ (methyl) and refined in a riding mode $\left[U_{\text {iso }}(\mathrm{H})=1.2 U_{\text {eq }} \mathrm{C}\right.$ (aromatic and methylene)], $1.5 U_{\text {eq }} \mathrm{C}$ (methyl).

\section{Comment}

Various ONNO ligands stabilizing Ti(IV) complexes containing different substituents have been synthesized and tested for antitumor activities in recent years [4]. The use of pyridine-2,6-dicarboxylate as a second chelator gives a novel heptacoordinated Ti(IV) complex with enhanced aqueous stability and cytotoxicity [5, 6], which is considered as a new generation of anti-tumor Ti complexes [7]. The isotopic titanium complex [45Ti][(Salan) $\mathrm{Ti}^{(\mathrm{IV})}$ (Dipic)] could be synthesized based on this ligand system and studied as PET probes [8, 9]. ONNO Ti(IV) complexes are structurally close to ONON Ti(IV) complexes, which exhibit much higher space flexibility because of the amino side arm [10]. However, only one report is related to the ONON Ti(IV) complex due to the difficult synthesis of ligands [10], no reports are found on the ONON Ti(IV) complexes containing a naphthalene moiety. 
As shown in the Figure, the central Ti cation is sevencoordinated by three nitrogen atoms and four oxygen atoms, forming a distorted pentagonal bipyramidal geometry. The bond lengths of four $\mathrm{Ti}-\mathrm{O}$ and three $\mathrm{Ti}-\mathrm{N}$ bonds are ranging from 1.8390(12) to 2.0902(13) $\AA$ and from 2.1906(14) to 2.3891(15) A, respectively, which is in accordance with the literature [7]. Surprisingly, the Mannich reaction of naphthalen-2-ol gave a rearranged ONON ligand $\mathrm{L}_{1}$, thus giving the titled ONON-Ti(IV) compound. The $\mathrm{CH}_{2} \mathrm{Cl}_{2}$ is disordered by an inversion center.

In the crystal packing, the molecules are linked together by some weak intermolecular interactions such as $\pi \cdots \pi$ and $\mathrm{C}-\mathrm{H} \cdots \pi$. For instance, the centroid-to-centroid distance between two inversion-related $\mathrm{C} 1-\mathrm{C} 6$ aromatic rings is only 3.596(1) $\AA$, indicating a $\pi \cdots \pi$ interaction.

Author contributions: All the authors have accepted responsibility for the entire content of this submitted manuscript and approved submission.

Research funding: Natural Science Foundation of Gansu Province of China (Nos. 20JR5RA470).

Conflict of interest statement: The authors declare no conflicts of interest regarding this article.

\section{References}

1. Bruker. SMART and SAINT for Windows NT Software Reference Manuals. version 5.0; Bruker Analytical X-Ray Systems: Madison, WI, 1997.
2. Sheldrick G. M. A short history of SHELX. Acta Crystallogr. 2008, A64, 112-122.

3. Slitikov P. V., Rasadkina E. N. Aminomethylation of naphthalen2-ol and naphthalene-2,7-diol. Russ. J. Org. Chem. 2016, 52, 1432-1435.

4. Immel T. A., Groth U., Huhn T. Cytotoxic titanium salan complexes: surprising Interaction of salan and alkoxy ligands. Chem. Eur J. 2010, 16, 2775-2789.

5. Grützke M., Zhao T., Immel T. A., Huhn T. Heptacoordinate heteroleptic salan (ONNO) and thiosalan (OSSO) titanium(IV) complexes: investigation of stability and cytotoxicity. Inorg. Chem. 2015, 54, 6697-6706.

6. Zhao T., Grützke M., Götz K. H., Druzhenko T., Huhn T. Synthesis and $\mathrm{X}$-ray structure analysis of cytotoxic heptacoordinate sulfonamide salan titanium(IV)-bis-Chelates. Dalton Trans. 2015, 44, 16475-16485.

7. Immel T. A., Grützke M., Späte A.-K., Groth U., Öhlschläger P., Huhn T. Synthesis and X-ray structure analysis of a heptacoordinate titanium(IV)-bis-chelate with enhanced in vivo antitumor efficacy. Chem. Commun. 2012, 48, 5790-5792.

8. Søborg Pedersen K., Baun C., Michaelsen Nielsen K., Thisgaard H., Ingemann Jensen A., Zhuravlev F. Design, synthesis, computational, and preclinical evaluation of natTi/45Tilabeled urea-based glutamate PSMA ligand. Molecules 2020, 52,1104

9. Severin G. W., Nielsen C. H., Jensen A. I., Fonslet J., Kjar A., Zhuravlev $F$. Bringing radiotracing to titanium-based antineoplastics: solid phase radiosynthesis, PET and ex vivo evaluation of antitumor agent $\left[{ }^{45} \mathrm{Ti}\right]($ salan)Ti(dipic). J. Med. Chem. 2015, 58, 7591-7595.

10. Peri D., Manna C. M., Shavit M., Tshuva E. Y. Ti complexes of branched diamine bis(phenolato) ligands: hydrolysis and cytotoxicity. Eur. J. Inorg. Chem. 2011, 2011, 4896-4900. 